\title{
PERIODIC ORBITS FOR MULTIVALUED MAPS WITH CONTINUOUS MARGINS OF INTERVALS
}

\author{
Jiehua MaI — TAIXIANG Sun
}

\begin{abstract}
Let $I$ be a bounded connected subset of $\mathbb{R}$ containing more than one point, and $\mathcal{L}(I)$ be the family of all nonempty connected subsets of $I$. Each map from $I$ to $\mathcal{L}(I)$ is called a multivalued map. A multivalued map $F: I \rightarrow \mathcal{L}(I)$ is called a multivalued map with continuous margins if both the left endpoint and the right endpoint functions of $F$ are continuous. We show that the well-known Sharkovskiu theorem for interval maps also holds for every multivalued map with continuous margins $F: I \rightarrow \mathcal{L}(I)$, that is, if $F$ has an $n$-periodic orbit and $n \succ m$ (in the Sharkovskii ordering), then $F$ also has an $m$-periodic orbit.
\end{abstract}

\section{Introduction}

Let $X$ be a set and $\mathbb{N}=\{1,2, \ldots\}$. An infinite sequence $\left(x_{1}, x_{2}, \ldots\right)$ of elements in $X$ is said to be periodic if there is $n \in \mathbb{N}$ such that

$$
x_{i+n}=x_{i} \quad \text { for all } i \in \mathbb{N} \text {. }
$$

In this case, we also write $\left(x_{1}, \ldots, x_{n}\right)^{\circ}$ for $\left(x_{1}, x_{2}, \ldots\right)$, where we put the small circle $\circ$ at the top-right corner of the finite sequence $\left(x_{1}, \ldots, x_{n}\right)$, which means that we repeat this finite sequence infinitely many times. The least $n$ such that (1.1) holds is called the period of $\left(x_{1}, x_{2}, \ldots\right)$. Note that if we cannot clearly

2010 Mathematics Subject Classification. 54C60, 54C05, 37E05, 37C27.

Key words and phrases. Multivalued map; interval map; periodic orbit; period; Sharkovskiǐ's order.

This project is supported by NNSF of China (11261005, 11461003). 
mention the period of the infinite sequence $\left(x_{1}, \ldots, x_{n}\right)^{\circ}$, then it may be a proper factor of $n$. A periodic sequence of period $n$ is also called an $n$-periodic sequence.

Denote by $2^{X}-\{\emptyset\}$ the family of all nonempty subsets of $X$. Each map from $X$ to $2^{X}-\{\emptyset\}$ is called a multivalued map on $X$. An infinite sequence $\left(x_{1}, x_{2}, \ldots\right)$ of elements in $X$ is called an orbit of $F: X \rightarrow 2^{X}-\{\emptyset\}$ if $x_{i+1} \in F\left(x_{i}\right)$ for all $i \in \mathbb{N}$. The sequence $\left(x_{1}, x_{2}, \ldots\right)$ is called a periodic orbit of $F$ if it is both a periodic sequence and an orbit of $F$. If $\mathcal{O}=\left(x_{1}, x_{2}, \ldots\right)=\left(x_{1}, \ldots, x_{n}\right)^{\circ}$ is an $n$ periodic orbit of $F$, then, for any $i \in \mathbb{N}$, the finite sequence $\left(x_{i}, x_{i+1}, \ldots, x_{i+n-1}\right)$ with length $n$ is called a periodic segment of the orbit $\mathcal{O}$. If $F: X \rightarrow 2^{X}-\{\emptyset\}$ is a multivalued map and $F$ contains only one element for each $x \in X$, then $F$ is a single-valued map from $X$ to $X$. Note that if $f: X \rightarrow X$ is a single-valued map, then any period segment of a periodic orbit of $f$ contains no repeating element, and if $F: X \rightarrow 2^{X}-\{\emptyset\}$ is a multivalued map, then a period segment of some periodic orbit of $F$ may contain repeating elements. This is a difference between single-valued maps and multivalued maps. Since there may appear repeating elements in a period segment when we study periodic orbits of multivalued maps, it will meet some additional trouble.

Let $I$ be a bounded connected subset of $\mathbb{R}$ containing more than one point, that is, $I$ is a closed interval, or an open interval, or a half-open interval. Denote by $\bar{I}$ the closure of $I$ in $\mathbb{R}$ and by $\mathcal{L}(I)$ the family of all nonempty connected subsets of $I$. Each map from $I$ to $\mathcal{L}(I)$ is called a connected-multivalued map on $I$. Obviously, for any connected-multivalued map $F: I \rightarrow \mathcal{L}(I)$, there exists a unique pair of functions $\alpha: I \rightarrow \bar{I}$ and $\beta: I \rightarrow \bar{I}$, called the left endpoint function and the right endpoint function of $F$, respectively, satisfying the following two conditions:

(i) $\alpha(x) \leq \beta(x)$ for any $x \in I$;

(ii) $(\alpha(x), \beta(x)) \subset F(x) \subset[\alpha(x), \beta(x)]$ for any $x \in I$.

If $\alpha(x)=\beta(x)$, then $F(x)=[\alpha(x), \beta(x)]=\{\alpha(x)\}$.

A connected-multivalued map $F: I \rightarrow \mathcal{L}(I)$ is said to be a multivalued map with continuous margins if both the left endpoint and the right endpoint functions of $F$ are continuous.

In 1964, Sharkovskiu found the following order relation in $\mathbb{N}$ :

$$
\begin{aligned}
3 \succ 5 \succ 7 \succ \ldots \succ & 3 \cdot 2 \succ 5 \cdot 2 \succ 7 \cdot 2 \succ \ldots \succ 3 \cdot 2^{2} \succ 5 \cdot 2^{2} \succ 7 \cdot 2^{2} \succ \ldots \\
\ldots & \succ 3 \cdot 2^{k} \succ 5 \cdot 2^{k} \succ 7 \cdot 2^{k} \succ \ldots \succ 2^{4} \succ 2^{3} \succ 2^{2} \succ 2 \succ 1,
\end{aligned}
$$

and proved the following theorem.

THEOREM 1.1 (Sharkovskiü's theorem, see [17]). Let $J$ be a connected subset of $\mathbb{R}$ and $f: J \rightarrow J$ be a single-valued continuous map. For any $m, n \in \mathbb{N}$ with $n \succ m$, if $f$ has an $n$-periodic orbit, then $f$ has an $m$-periodic orbit. 
Note that the above Sharkovski1's order is well-ordered. If $n \succ m$ in this order, then we also write $m \prec n$.

In [1], Alseda and Llibre showed that Theorem 1.1 holds for triangular maps on a rectangle. In [12], Minc and Transue showed that Sharkovskii's theorem also holds for continuous maps on hereditarily decomposable chainable continua. In [5], Andres et al. also obtained a full analogy of Sharkovskiiı's theorem for lower-semicontinuous maps (i.e. for every closed subset $V \subset \mathbb{R}$, the set $\{x \in \mathbb{R}$ : $F(x) \subset V\}$ is closed) with nonempty, connected and compact values.

Recently, there has been a lot of work on the dynamics of multivalued maps (see [11], [13]-[16]). In [3], Andres et al. studied the periodic orbits of a class of multivalued maps and obtained the following theorem.

THEOREM 1.2. Let $\mathcal{C}(\mathbb{R})$ be the family of all nonempty compact connected subsets of $\mathbb{R}$ and $F: \mathbb{R} \rightarrow \mathcal{C}(\mathbb{R})$ be upper-semicontinuous (i.e. for every open $V \subset \mathbb{R}$, the set $\{x \in \mathbb{R}: F(x) \subset V\}$ is open). If $F$ has an n-periodic orbit for some odd integer $n$, but $F$ has no l-periodic orbit for any $l \succ n$, then for any $n \succ m, F$ has an $m$-periodic orbit, except $m=4$.

Further, Andres and Pastor [9] (also see [10]) obtained the following theorem.

TheOREM 1.3. Let $F: \mathbb{R} \rightarrow \mathcal{C}(\mathbb{R})$ be upper-semicontinuous. For any $m, n \in$ $\mathbb{N}$ with $n \succ m$, if $F$ has an n-periodic orbit, then $F$ has an m-periodic orbit with at most two exceptions.

For some other papers in the area, see also [2], [4], [6]-[8] and the references therein. In this paper, we study connected-multivalued maps on the bounded connected set $I$. Our main result is the following theorem.

TheOREM 1.4. Let I be a bounded connected subset of $\mathbb{R}$ and $F: I \rightarrow \mathcal{L}(I)$ be a multivalued map with continuous margins. For any $m, n \in \mathbb{N}$ with $n \succ m$, if $F$ has an n-periodic orbit, then $F$ has an m-periodic orbit.

REMARK 1.5. In [2]-[10], the set of every value of upper-semicontinuous and lower-semicontinuous maps is nonempty, it is a connected and compact set. But for multivalued maps with continuous margins of intervals studied in this paper, the set of every value need not be compact.

REMARK 1.6. In [3], the authors constructed upper-semicontinuous maps $F: \mathbb{R} \rightarrow \mathcal{C}(\mathbb{R})$ and $G: \mathbb{R} \rightarrow \mathcal{C}(\mathbb{R})$ such that $F$ has a 3-periodic orbit but has no 2-periodic orbit and $G$ has a 5-periodic orbit but has no 4-periodic orbit. While for multivalued maps with continuous margins of intervals studied in this paper, Sharkovskiîs theorem holds, without exception. 
ExAmple 1.7. Define a connected-multivalued map $F:[0,1] \rightarrow \mathcal{L}([0,1])$ by

$$
F(x)= \begin{cases}{[0,0]} & \text { if } x=0, \\ {[0, \sqrt{2} x)} & \text { if } x \in(0, \sqrt{2} / 2), \\ {[0,1]} & \text { if } x=\sqrt{2} / 2, \\ {[0,(2+\sqrt{2})(1-x))} & \text { if } x \in(\sqrt{2} / 2,0), \\ {[0,0]} & \text { if } x=1,\end{cases}
$$

$x \in[0,1]$. Then, according to our definition, $F$ is a multivalued map with continuous margins. But according to the definitions in [2]-[4], [6]-[10], $F$ is not upper-semicontinuous since the set $\{x \in[0,1]: F(x) \subset[0, y)\}=[0, y / \sqrt{2}] \cup$ $[1-y /(2+\sqrt{2}), 1]$ is closed for any $y \in(0,1]$. What means that continuity of margins does not necessarily implies upper-semicontinity of the multivalued map with continuous margins under consideration.

\section{Periodic orbits for multivalued maps}

Let $X$ be a set. Let $F$ and $G$ be maps from $X$ to $2^{X}-\{\emptyset\}$. Define the composite map $G \circ F: X \rightarrow 2^{X}-\{\emptyset\}$ by

$$
G \circ F(x)=\bigcup\{G(y): y \in F(x)\},
$$

$x \in X$. Denote by $F^{0}$ the identity map on $X, F^{1}=F$, and $F^{n+1}=F \circ F^{n}$ for each $n \in \mathbb{N}$. For $n \geq 0, F^{n}$ is called the $n$-th iterate of $F$.

REMARK 2.1. We see from the definition that for any $n \geq 2$ and any $x \in X$,

$$
\begin{aligned}
& F^{n}(x)=\left\{y \in X: \text { there exists }\left\{x_{i}\right\}_{i=0}^{n} \subset X\right. \\
& \text { such that } \left.x_{0}=x, x_{n}=y, x_{i} \in F\left(x_{i-1}\right) \text { for } 1 \leq i \leq n\right\} .
\end{aligned}
$$

Let $S=\left(x_{1}, x_{2}, \ldots\right)$ be an infinite sequence. For any $k, i \in \mathbb{N}$, the sequence

$$
\left(x_{i}, x_{k+i}, x_{2 k+i}, x_{3 k+i}, \ldots\right)
$$

is called the $i$-th $k$-subsequence of $S$. Obviously, if the sequence $S$ is an orbit of $F: X \rightarrow 2^{X}-\{\emptyset\}$, then any $k$-subsequence of $S$ is an orbit of $F^{k}$.

The following lemma is well-known, but we still give a simplified proof.

Lemma 2.2. Suppose that $\left(x_{1}, x_{2}, \ldots\right)$ is an infinite sequence. Let $n, m \in \mathbb{N}$ and $k=\operatorname{gcd}(n, m)$ be the greatest common factor of $n$ and $m$. If $x_{i+n}=x_{i}$ and $x_{i+m}=x_{i}$ for all $i \in \mathbb{N}$, then $x_{i+k}=x_{i}$ for all $i \in \mathbb{N}$.

Proof. As $k=\operatorname{gcd}(n, m)$, there exist $p, q \in \mathbb{N}$ such that $p n-q m=k$. Then we have $x_{i}=x_{i+p n}=x_{i+p n-q m}=x_{i+k}$ for any $i \in \mathbb{N}$.

Corollary 2.3. If $\left(x_{1}, x_{2}, \ldots\right)$ is a periodic sequence, which can be written as $\left(x_{1}, \ldots, x_{n}\right)^{\circ}$, then the period of this sequence is a factor of $n$. 
Definition 2.4. Two positive integers $k$ and $n$ are said to have the same prime factor if for any prime number $p, p$ is a factor of $k$ if and only if $p$ is a factor of $n$.

The following lemma is trivial.

Lemma 2.5. Suppose that integers $k$ and $n$ have the same prime factor. Then:

(a) $k=1$ if and only if $n=1$.

(b) If $k>1$, then there exist prime numbers $p_{1}, \ldots, p_{m}$ with $m \geq 1$ and positive integers $\lambda_{1}, \ldots, \lambda_{m}, \mu_{1}, \ldots, \mu_{m}$ such that

$$
k=\prod_{i=1}^{m} p_{i}^{\lambda_{i}} \quad \text { and } \quad n=\prod_{i=1}^{m} p_{i}^{\mu_{i}} .
$$

Lemma 2.6. Let $k, n \in \mathbb{N}$. Then there exists a unique sequence $\left(k_{1}, k_{2}, n_{1}, n_{2}\right)$ of positive integers such that

(a) $k=k_{1} k_{2}$ and $n=n_{1} n_{2}$,

(b) $k_{1}$ and $n_{1}$ have the same prime factor,

(c) $\operatorname{gcd}\left(k_{2}, n\right)=1$ and $\operatorname{gcd}\left(n_{2}, k\right)=1$.

Proof. Let $k_{2}=\max \{\lambda: \lambda$ is a factor of $k$ and $\operatorname{gcd}(\lambda, n)=1\}$ and $n_{2}=$ $\max \{\mu: \mu$ is a factor of $n$ and $\operatorname{gcd}(\mu, k)=1\}$. Put $k_{1}=k / k_{2}$ and $n_{1}=n / n_{2}$. Then the sequence $\left(k_{1}, k_{2}, n_{1}, n_{2}\right)$ satisfies three conditions in Lemma 2.6. Moreover, it is easy to show that the sequence $\left(k_{1}, k_{2}, n_{1}, n_{2}\right)$ satisfying these three conditions is unique, so the process can be omitted.

The main result in this section is the following lemma.

Lemma 2.7. Suppose that $S=\left(x_{1}, x_{2}, \ldots\right)=\left(x_{1}, \ldots, x_{n k}\right)^{\circ}$ is a periodic sequence with $n>1$ and $k>1$, and $S_{k}=\left(x_{1}, x_{k+1}, x_{2 k+1}, \ldots, x_{(n-1) k+1}\right)^{\circ}$ is a $k$-subsequence of $S$. Let $\left(k_{1}, k_{2}, n_{1}, n_{2}\right)$ be the same as in Lemma 2.6. If the period of $S_{k}$ is $n$, then there is a factor $\lambda$ of $k_{2}$ such that the period of $S$ is $k_{1} \lambda n$.

Proof. Let $m$ be the period of the sequence $S$. According to Corollary 2.3, $m$ is a factor of $k n$. Then $\operatorname{gcd}(m, k n)=m$. Write $n_{3}=\operatorname{gcd}\left(m, n_{2}\right)$. Then $n_{1} n_{3}$ is a factor of $n=n_{1} n_{2}$. As $\operatorname{gcd}\left(n_{2}, k n_{1}\right)=1$, we have $m=\operatorname{gcd}(m, k n)=$ $\operatorname{gcd}\left(m, k n_{1} n_{2}\right)=\operatorname{gcd}\left(m, k n_{1}\right) \cdot \operatorname{gcd}\left(m, n_{2}\right)=\operatorname{gcd}\left(m, k n_{1}\right) \cdot n_{3}$. Hence $m$ is a factor of $k n_{1} n_{3}$, which implies

$$
x_{i+k n_{1} n_{3}}=x_{i} \quad \text { for all } i \in \mathbb{N} .
$$

On the other hand, if $n_{1} n_{3}<n$, then $x_{j+k n_{1} n_{3}}=x_{j}$ does not hold for some $j \in\{1, k+1,2 k+1, \ldots,(n-1) k+1\}$ since the period of the sequence $S_{k}$ is $n$. This will contradict to (2.2). Thus we must have $n_{1} n_{3}=n$, which means that $n_{2}=n_{3}=\operatorname{gcd}\left(m, n_{2}\right)$. Hence we obtain:

Claim 1. $m=n_{2} r$ for some $r \in \mathbb{N}$. 
Let $k_{3}=\operatorname{gcd}\left(m, k_{1} n_{1}\right)$. As $\operatorname{gcd}\left(k_{1} n_{1}, k_{2} n_{2}\right)=1$, we have

$$
\begin{aligned}
m & =\operatorname{gcd}(m, k n)=\operatorname{gcd}\left(m, k_{1} n_{1} \cdot k_{2} n_{2}\right) \\
& =\operatorname{gcd}\left(m, k_{1} n_{1}\right) \cdot \operatorname{gcd}\left(m, k_{2} n_{2}\right)=k_{3} \cdot \operatorname{gcd}\left(m, k_{2} n_{2}\right) .
\end{aligned}
$$

Let $k_{4}=\operatorname{lcm}\left(k_{1}, k_{3}\right)$ be the least common multiple of $k_{1}$ and $k_{3}$. Then $m$ is a factor of $k_{4} k_{2} n_{2}$, and hence

$$
x_{i}=x_{i+k_{4} k_{2} n_{2}} \quad \text { for all } i \in \mathbb{N} .
$$

On the other hand, if $k_{3}$ is a proper factor of $k_{1} n_{1}$, then $k_{1}>1, n_{1}>1$, and from the condition (b) of Lemma 2.6, we see that $k_{4}$ is also a proper factor of $k_{1} n_{1}$, which implies that $k_{4} k_{2} n_{2}$ is a proper factor of $n k=k_{1} n_{1} k_{2} n_{2}$. Thus there is a proper factor $n_{4}$ of $n$ such that $k_{4} k_{2} n_{2}=k n_{4}$. However, $x_{j+k n_{4}}=x_{j}$ does not hold for some $j \in\{1, k+1,2 k+1, \ldots,(n-1) k+1\}$ since the period of the sequence $S_{k}$ is $n$. This will contradict to (2.3). Thus we must have $k_{3}=\operatorname{gcd}\left(m, k_{1} n_{1}\right)=k_{1} n_{1}$ and hence we obtain

Claim 2. $m=k_{1} n_{1} r$ for some $r \in \mathbb{N}$.

As $\operatorname{gcd}\left(k_{1} n_{1}, n_{2}\right)=1$, by Claims 1 and 2 , we see that $m=k_{1} n_{1} n_{2} r=k_{1} n r$ for some $r \in \mathbb{N}$. Hence there exists a factor $\lambda$ of $k_{2}$ such that $m=k_{1} \lambda n$ since $m$ is a factor of $k n=k_{1} k_{2} n_{1} n_{2}$.

Conversely, we have

Lemma 2.8. Let $k, n$ and $\left(k_{1}, k_{2}, n_{1}, n_{2}\right)$ be the same as in Lemma 2.6. Then, for any factor $\lambda$ of $k_{2}$, there exists a $k_{1} \lambda n$-periodic sequence $S=\left(x_{1}, x_{2}, \ldots\right)=$ $\left(x_{1}, \ldots, x_{k n}\right)^{\circ}$ such that the period of the $k$-subsequence $S_{k}=\left(x_{1}, x_{k+1}, x_{2 k+1}, \ldots\right.$, $\left.x_{(n-1) k+1}, \ldots\right)$ is $n$.

Proof. Let $m=k_{1} \lambda n$. Then $m$ is a factor of $k n$. Take an $m$-periodic sequence $S=\left(x_{1}, x_{2}, \ldots\right)=\left(x_{1}, \ldots, x_{m}\right)^{\circ}$ such that $x_{1}, \ldots, x_{m}$ are pairwise different elements. Noting that $x_{i+m}=x_{i}$ for all $i \in \mathbb{N}$, we can also write $S=\left(x_{1}, \ldots, x_{k n}\right)^{\circ}$. For $0 \leq i<j \leq n-1$, we have $(j-i) k_{2} / n \notin \mathbb{N}$ since $\operatorname{gcd}\left(k_{2}, n\right)=1$, which implies that $(j-i) k /\left(k_{1} \lambda n\right)=(j-i) k_{2} /(\lambda n) \notin \mathbb{N}$, and hence $j k+1 \not \equiv i k+1\left(\bmod k_{1} \lambda n\right)$. Thus $x_{1}, x_{k+1}, x_{2 k+1}, \ldots, x_{(n-1) k+1}$ are pairwise different elements, and hence the period of $S_{k}$ is $n$.

Lemma 2.9. Suppose that $S=\left(x_{1}, x_{2}, \ldots\right)=\left(x_{1}, \ldots, x_{k n}\right)^{\circ}$ is a kn-periodic sequence with $k \geq 2$ and $n \geq 2$. Let $S_{i}=\left(x_{i}, x_{k+i}, x_{2 k+i}, \ldots, x_{(n-1) k+i}\right)^{\circ}$, for each $i \in \mathbb{N}$, be the $i$-th $k$-subsequence of $S$. Then:

(a) There exists $i \in\{1, \ldots, k\}$ such that the period of $S_{i}$ is a factor of $n$ greater than 1.

(b) If there exist a prime number $p$ and $\lambda \in \mathbb{N}$ such that $n=p^{\lambda}$, then there exists $i \in\{1, \ldots, k\}$ such that the period of $S_{i}$ is $n$. 
Proof. Since the length of the finite sequence $\left(x_{i}, x_{k+i}, x_{2 k+i}, \ldots, x_{(n-1) k+i}\right)$ is $n$, by Corollary 2.3, the period of $S_{i}$ must be a factor of $n$.

(a) is obvious, since, otherwise, if for each $i \in\{1, \ldots, k\}$, the period of $S_{i}$ is 1, then the period of $S$ will be a factor of $k$, which contradicts the condition of the lemma that period of $S$ is $k n$.

(b) is also obvious, since, otherwise, if for each $i \in\{1, \ldots, k\}$, the period of $S_{i}$ is a proper factor of $n=p^{\lambda}$, then the period of $S$ will be a proper factor of $k n$, which also contradicts the condition of the lemma.

REMARK 2.10. In Lemma 2.9, if $n$ is not an integral power of some prime number, then it is possible that the period of any $k$-subsequence of $S$ is a proper factor of $n$. For example, let $k=2, n=6$, and let $x_{1}, x_{2}, y_{1}, y_{2}, y_{3}$ be pairwise different elements. Then the period of any 2 -subsequence of the 12-periodic sequence $S=\left(x_{1}, y_{1}, x_{2}, y_{2}, x_{1}, y_{3}, x_{2}, y_{1}, x_{1}, y_{2}, x_{2}, y_{3}\right)^{\circ}$ is a proper factor of 6 .

From Lemma 2.7 we get

Corollary 2.11. Suppose that $X$ is a set and $F: X \rightarrow 2^{X}-\{\emptyset\}$ is a multivalued map. Let $k, n$ and $\left(k_{1}, k_{2}, n_{1}, n_{2}\right)$ be the same as in Lemma 2.6. If $F^{k}$ has an n-periodic orbit, then $F$ itself has a periodic orbit, of which the period is a factor of $k n$ and is an integral multiple of $k_{1} n$.

Proof. Let $O_{k}=\left(x_{1}, x_{k+1}, x_{2 k+1}, \ldots, x_{(n-1) k+1}\right)^{\circ}$ be an $n$-periodic orbit of $F^{k}$. By Remark 2.1, $O_{k}$ can be extended to be a periodic orbit

$$
O=\left(x_{1}, \ldots, x_{k}, x_{k+1}, \ldots, x_{2 k}, x_{2 k+1}, \ldots, x_{(n-1) k+1}, \ldots, x_{n k}\right)^{\circ}
$$

of $F$. By Lemma 2.7, the period of $O$ is $k_{1} \lambda n$ for some factor $\lambda$ of $k_{2}$.

From Lemma 2.9 we get the following corollary at once.

Corollary 2.12. Let $F: X \rightarrow 2^{X}-\{\emptyset\}$ be a multivalued map. Suppose that $F$ has a kn-periodic orbit $O=\left(x_{1}, x_{2}, \ldots\right)=\left(x_{1}, \ldots, x_{k n}\right)^{\circ}$ with $k \geq 2$ and $n \geq 2$. Then:

(a) The $k$-th iterate $F^{k}$ has a periodic orbit, whose period is a factor of $n$ greater than 1.

(b) If there exist a prime number $p$ and $\lambda \in \mathbb{N}$ such that $n=p^{\lambda}$, then $F^{k}$ has an n-periodic orbit.

\section{Multivalued maps with continuous margins of intervals}

Let $I$ be a bounded connected subset of $\mathbb{R}$. Recall that each map $F: I \rightarrow \mathcal{L}(I)$ is called a connected-multivalued map on $I$, and $F$ is a multivalued map with continuous margins if both the left endpoint $\alpha: I \rightarrow \bar{I}$ and the right endpoint functions $\beta: I \rightarrow \bar{I}$ of $F$ are continuous. 
Lemma 3.1. Let $F: I \rightarrow \mathcal{L}(I)$ and $G: I \rightarrow \mathcal{L}(I)$ be multivalued maps with continuous margins. Then the composite function $G \circ F$ also is a multivalued map with continuous margins from $I$ to $\mathcal{L}(I)$.

Proof. Let $\alpha_{1}, \beta_{1}$ and $\alpha_{2}, \beta_{2}$ be the left endpoint and right endpoint functions of $F$ and $G$, respectively. Define $\alpha_{3}: I \rightarrow \bar{I}$ and $\beta_{3}: I \rightarrow \bar{I}$ by

$$
\alpha_{3}(x)=\inf \left\{\alpha_{2}(y): y \in F(x)\right\} \quad \text { and } \quad \beta_{3}(x)=\sup \left\{\beta_{2}(y): y \in F(x)\right\},
$$

$x \in I$. For any $u, v \in \mathbb{R}$, denote by $\langle u, v\rangle$ the smallest connected subset containing $u$ and $v$ in $\mathbb{R}$. Then we have

$$
\left(\alpha_{3}(x), \beta_{3}(x)\right) \subset G \circ F(x) \subset\left[\alpha_{3}(x), \beta_{3}(x)\right]
$$

since $F(x)$ is connected and $\alpha_{2}$ is continuous. It is easy to see that for any $x, w \in I,\left|\alpha_{3}(w)-\alpha_{3}(x)\right| \leq \max \left\{S_{1}, S_{2}\right\}$, where

$$
\begin{aligned}
& S_{1}=\sup \left\{\alpha_{2}(u)-\alpha_{2}(v):\{u, v\} \subset\left\langle\alpha_{1}(x), \alpha_{1}(w)\right\rangle \cap I\right\}, \\
& S_{2}=\sup \left\{\alpha_{2}(u)-\alpha_{2}(v):\{u, v\} \subset\left\langle\beta_{1}(x), \beta_{1}(w)\right\rangle \cap I\right\} .
\end{aligned}
$$

Noting that $\alpha_{1}, \beta_{1}$ and $\alpha_{2}$ are continuous, we derive that $\alpha_{3}(w) \rightarrow \alpha_{3}(x)$ as $w \rightarrow x$. Thus $\alpha_{3}$ is continuous. In a similar fashion, we can show that $\beta_{3}$ is also continuous. Hence $G \circ F$ is a multivalued map with continuous margins from $I$ to $\mathcal{L}(I)$.

Definition 3.2. Let $F: X \rightarrow 2^{X}-\{\emptyset\}$ be a multivalued map, and $f: X \rightarrow X$ be a single-valued map. We say that $F$ contains $f$ or $f$ is contained by $F$ if $f(x) \in F(x)$ for any $x \in X$. If $f$ is contained by $F$, then we write $f \dot{\in} F$.

The following is one of the key lemmas in this paper.

LEMMA 3.3. Let $F: I \rightarrow \mathcal{L}(I)$ be a multivalued map with continuous margins and $n \in \mathbb{N}$. Then for any pairwise different points $x_{1}, \ldots, x_{n}$ in $I$ and any given $y_{i} \in F\left(x_{i}\right), 1 \leq i \leq n$, there exists a continuous map $f: I \rightarrow I$ such that $f \dot{\in} F$ and $f\left(x_{i}\right)=y_{i}$ for every $1 \leq i \leq n$.

Proof. Let $\alpha$ and $\beta$ be the left endpoint and right endpoint functions of $F$, respectively. For any $i \in\{1, \ldots, n\}$, obviously, there is a real number $t_{i} \in[0,1]$ such that $y_{i}=t_{i} \alpha\left(x_{i}\right)+\left(1-t_{i}\right) \beta\left(x_{i}\right)$. Take a continuous function $t: I \rightarrow[0,1]$ such that

$$
\begin{array}{ll}
t\left(x_{i}\right)=t_{i} & \text { for } i \in\{1, \ldots, n\} \\
t(x) \in(0,1) & \text { for any } x \in I-\left\{x_{1}, \ldots, x_{n}\right\} .
\end{array}
$$

Define $f: I \rightarrow I$ by

$$
f(x)=t(x) \cdot \alpha(x)+(1-t(x)) \cdot \beta(x) \quad \text { for any } x \in I .
$$


Then $f$ is continuous. By (3.1) and (3.3), we have $f\left(x_{i}\right)=y_{i}$ for $i \in\{1, \ldots, n\}$. By (3.2) and (3.3), we get

$$
f(x) \in(\alpha(x), \beta(x)) \subset F(x) \text { for any } x \in I-\left\{x_{1}, \ldots, x_{n}\right\} .
$$

Thus $f \dot{\in} F$.

From Lemma 3.3 we obtain the following corollary at once.

Corollary 3.4. Let $F: I \rightarrow \mathcal{L}(I)$ be a multivalued map with continuous margins, and $O=\left(x_{1}, x_{2}, \ldots\right)=\left(x_{1}, \ldots, x_{n}\right)^{\circ}$ be an $n$-periodic orbit of $F$, where $n \in \mathbb{N}$. If $x_{1}, \ldots, x_{n}$ are pairwise different, then $F$ contains a continuous map $f: I \rightarrow I$ such that $O=\left(x_{1}, \ldots, x_{n}\right)^{\circ}$ is also an n-periodic orbit of $f$, and hence, for any $m \in \mathbb{N}$ with $n \succ m, f$ and $F$ have an m-periodic orbit.

If $\left(x_{1}, x_{2}, \ldots\right)=\left(x_{1}, x_{2}\right)^{\circ}$ is a 2 -periodic sequence, then we must have $x_{1} \neq$ $x_{2}$. Therefore, from Corollary 3.4 we get

COROLlary 3.5. If a multivalued map with continuous margins $F: I \rightarrow \mathcal{L}(I)$ has a 2-periodic orbit, then $F$ has a 1-periodic orbit.

Corollary 3.6. Let $F: I \rightarrow \mathcal{L}(I)$ be a multivalued map with continuous margins. If $F$ has a 3-periodic orbit $\left(x_{1}, x_{2}, \ldots\right)=\left(x_{1}, x_{2}, x_{3}\right)^{\circ}$, then $F$ has an $m$-periodic orbit for any $m \in \mathbb{N}$.

Proof. By Corollary 3.4, we can consider only the case that $x_{i}=x_{j}$ for some $1 \leq i<j \leq 3$, that is, there exists $k \in\{1,2,3\}$ such that $x_{k}=x_{k+1} \neq$ $x_{k+2}$. From this we see that $F$ has a 1-periodic orbit $\left(x_{k}\right)^{\circ}$, a 2-periodic orbit $\left(x_{k}, x_{k+2}\right)^{\circ}$, and an $m$-periodic orbit $\left(x_{k}, x_{k+2}, y_{1}, \ldots, y_{m-2}\right)^{\circ}$ for any $m \geq 3$, where $y_{1}=\ldots=y_{m-2}=x_{k}$.

Corollary 3.7. Let $F: I \rightarrow \mathcal{L}(I)$ be a multivalued map with continuous margins. If $F$ has a 4-periodic orbit $\left(x_{1}, x_{2}, \ldots\right)=\left(x_{1}, x_{2}, x_{3}, x_{4}\right)^{\circ}$, then $F$ has a 2-periodic orbit.

Proof. By Corollary 3.4, we can consider only the case that $x_{i}=x_{j}$ for some $1 \leq i<j \leq 5$ with $i \leq 4$ and $j \leq i+2$. If $j=i+1$, then $F$ has a 3 -periodic orbit $\left(x_{j+1}, x_{j+2}, x_{j+3}\right)^{\circ}$, and hence has a 2 -periodic orbit. If $j=i+2$, then at least one of the two orbits $\left(x_{i}, x_{i+1}\right)^{\circ}$ and $\left(x_{j}, x_{j+1}\right)^{\circ}$ is a 2-periodic orbit.

Lemma 3.8. Let $F: I \rightarrow \mathcal{L}(I)$ be a multivalued map with continuous margins. If $F$ has a $2^{\lambda}$-periodic orbit, then $F$ has a $2^{\lambda-1}$-periodic orbit.

Proof. It follows from Corollaries 3.5 and 3.7 that Lemma 3.8 holds for the case that $\lambda \in\{1,2\}$. In what follows we can assume that $\lambda \geq 3$. By (b) of Corollary 2.12, we see that $F^{2^{\lambda-2}}$ has a 4 -periodic orbit. This combining with Corollary 3.7 implies that $F^{2^{\lambda-2}}$ has a 2-periodic orbit. Using Corollary 2.11 
in the case that $k=k_{1}=2^{\lambda-2}$ and $n=2$, we see that $F$ has a $2^{\lambda-1}$-periodic orbit.

Now we give the main result of this paper and its proof.

TheOREM 3.9. Let $I$ be a bounded connected subset of $\mathbb{R}$ and $F: I \rightarrow \mathcal{L}(I)$ be a multivalued map with continuous margins. For any $m, n \in \mathbb{N}$ with $n \succ m$, if $F$ has an n-periodic orbit, then $F$ has an m-periodic orbit.

Proof. If $x_{1}, \ldots, x_{n}$ are pairwise different points, then by Corollary 3.4, we see that Theorem 3.9 holds. We can add the following hypothesis:

$\left(\mathrm{H}_{1}\right)$ There exist $1 \leq i<j \leq i+n-2$ such that $x_{i}=x_{j} \neq x_{j+1}$ and $j-i$ is the least, that is, if there exist $1 \leq i^{\prime}<j^{\prime} \leq i^{\prime}+n-2$ such that $x_{i^{\prime}}=x_{j^{\prime}} \neq x_{j^{\prime}+1}$, then $j^{\prime}-i^{\prime} \geq j-i$. Further, we may assume that $x_{j+1}>x_{j}$.

By Lemmas 3.6 and 3.8, we can add the following hypothesis:

$\left(\mathrm{H}_{2}\right)$ For any $\lambda \in \mathbb{N}, 3 \succ n \succ 2^{\lambda}$, and it has been proved that, for any $n_{0} \in \mathbb{N}$ with $3 \succeq n_{0} \succ n$ and for any multivalued map with continuous margins $G: I \rightarrow \mathcal{L}(I)$, if $G$ has an $n_{0}$-periodic orbit, then for any $m \in \mathbb{N}$ with $n_{0} \succ m, G$ has an $m$-periodic orbit.

There are three cases to be considered.

Case 1. $n>3$ is odd and $j-i \geq 2$.

In this case, by $\left(\mathrm{H}_{1}\right), O_{1} \equiv\left(x_{i}, \ldots, x_{j-1}\right)^{\circ}$ and $O_{2} \equiv\left(x_{j}, \ldots, x_{i+n-1}\right)^{\circ}$ are also periodic orbits of $F$, whose periods are greater than 1 and are factors of $j-i$ and $i+n-j$, respectively. Hence, since one of the integers $j-i$ and $i+n-j$ is odd, $F$ has an $n_{0}$-periodic orbit for some odd $n_{0}$ with $3 \succeq n_{0} \succ n$. Therefore, by $\left(\mathrm{H}_{2}\right)$, for any $m \in \mathbb{N}$ with $n \succ m, F$ has an $m$-periodic orbit.

Case 2. $n>3$ is odd and $j-i=1$.

There are two subcases.

Subcase 2.1. There is $k \in\{3, \ldots, n-1\}$ such that $x_{i+k}=x_{i}$. In this subcase, $O_{1} \equiv\left(x_{i}, \ldots, x_{i+k-1}\right)^{\circ}$ and $O_{2} \equiv\left(x_{i+1}, \ldots, x_{i+k-1}\right)^{\circ}$ are periodic orbits of $F$, whose periods are greater than 1 and are factors of $k$ and $k-1$, respectively. Since one of the integers $k$ and $k-1$ is odd, similar to Case 1 , for any $m \in \mathbb{N}$ with $n \succ m, F$ has an $m$-periodic orbit.

Subcase 2.2. $x_{i+\lambda} \neq x_{i}$ for any $\lambda \in\{2, \ldots, n-1\}$. In this subcase, there is $k \in\{2, \ldots, n-1\}$ such that $x_{i+k+1} \leq x_{i}$ and $x_{i+\lambda}>x_{i}$ for $\lambda \in\{2, \ldots, k\}$. Let $Z_{0}=\left\{\lambda: \lambda \in\{2, \ldots, k\}\right.$ and $\left.x_{i+\lambda} \geq x_{i+k}\right\}$. Then $k \in Z_{0}$. Let $q=\min Z_{0}$. If $q>2$, then $x_{i}<x_{i+q-1}<x_{i+k} \leq x_{i+q}$. By Lemma 3.3, $F$ contains a continuous map $f: I \rightarrow I$ such that $f\left(x_{i}\right)=x_{i}, f\left(x_{i+q-1}\right)=x_{i+q} \geq x_{i+k}$ and $f\left(x_{i+k}\right)=$ $x_{i+k-1} \leq x_{1}$. Thus $f$ is turbulent since $f\left(\left[x_{i}, x_{i+q-1}\right]\right) \cap f\left(\left[x_{i+q-1}, x_{k}\right]\right) \supset\left[x_{i}, x_{k}\right]$. 
It is well-known that a turbulent interval map, $f$ (and hence $F$ ), has an $m$ periodic orbit for any $m \in \mathbb{N}$.

If $q=2$, then $x_{i+k} \in\left(x_{i}, x_{i+2}\right] \subset F\left(x_{i}\right)$. By Lemma 3.3, $F$ contains a continuous map $f: I \rightarrow I$ such that $f\left(x_{i}\right)=x_{i+2} \geq x_{i+k}>x_{i}$ and $f\left(x_{i+k}\right)=x_{i+k+1} \leq$ $x_{i}$, which implies that there is a point $y \in\left(x_{i}, x_{i+k}\right]$ such that $f(y)=x_{i}$, and hence $F$ has a 3 -periodic orbit $\left(x_{i}, x_{i}, y\right)^{\circ}$. By Lemma 3.6, $F$ has an $m$-periodic orbit for any $m \in \mathbb{N}$.

Case 3. $n=2^{\lambda}(2 \mu+1)$ for some $\lambda, \mu \in \mathbb{N}$.

In this case, from (a) of Corollary 2.12 we see that $F^{2^{\lambda}}$ has a periodic orbit which period is a factor of $2 \mu+1$ greater than 1 . By Lemma 3.8, we may assume that $n \succ m \succ 2^{\lambda}$.

If $n \succ m \succ 3 \cdot 2^{\lambda+1}$, then there is $\mu_{0} \in \mathbb{N}$ such that $m=2^{\lambda}\left(2 \mu+2 \mu_{0}+1\right)$. By hypothesis $\left(\mathrm{H}_{2}\right), F^{2^{\lambda}}$ has a $\left(2 \mu+2 \mu_{0}+1\right)$-periodic orbit. By Corollary 2.11, there is a factor $k_{2}$ of $2^{\lambda}$ such that $F$ has a $k_{2}\left(2 \mu+2 \mu_{0}+1\right)$-periodic orbit $O_{m}$. If $k_{2}=2^{\lambda}, O_{m}$ itself is an $m$-periodic orbit of $F$. If $k_{2}$ is a proper factor of $2^{\lambda}$, then $3 \succeq k_{2}\left(2 \mu+2 \mu_{0}+1\right) \succ n$, and from $\left(\mathrm{H}_{2}\right)$ we see that $F$ has an $m$-periodic orbit.

If $3 \cdot 2^{\lambda+1} \succeq m \succ 2^{\lambda}$, then there is $m_{0} \in \mathbb{N}$ such that $m=2^{\lambda} \cdot 2 m_{0}$. By hypothesis $\left(\mathrm{H}_{2}\right), F^{2^{\lambda}}$ has a $2 m_{0}$-periodic orbit. Using Corollary 2.11 to the case that $k=k_{1}=2^{\lambda}$, we see that $F$ has an $m$-periodic orbit.

\section{REFERENCES}

[1] L. Alseda And J. Llibre, Periods for triangular maps, Bull. Austral. Math. Soc. 47 (1993), 41-53.

[2] J. Andres, Period two implies chaos for a class of multivalued maps: A naive approach, Comput. Math. Appl. 64 (2012), 2160-2165.

[3] J. ANDRES, J. FIŠER AND L. JüTtNeR, On a multivalued version of the Sharkovskǐ theorem and its application to differential inclusions, Set-Valued Anal. 10 (2002), 1-14.

[4] J. Andres, T. Fürst And K. Pastor, Period two implies all periods for a class of ODEs: a multivalued map approach, Proc. Amer. Math. Soc. 135 (2007), 3187-3191.

[5] _ Full analogy of Sharkovskii's theorem for lower semicontinuous maps, J. Math. Anal. Appl. 340 (2008), 1132-1144.

[6] _ Sharkovski乞's theorem, differential inclusions, and beyond, Topol. Methods Nonlinear Anal. 33 (2009), 149-168.

[7] J. Andres, L. JÜttner And K. Pastor, On a multivalued version of the Sharkovskiน theorem and its application to differential inclusions II, Set-Valued Anal. 13 (2005), 4768.

[8] J. Andres And K. Pastor, On a multivalued version of the Sharkovskii theorem and its application to differential inclusions III, Topol. Methods Nonliear. Anal. 22 (2003), 369-386.

[9] A version of Sharkovskiu's theorem for differential equations, Proc. Amer. Math. Soc. 133 (2004), 449-453.

[10] J. Andres, K. Pasto And P. ŠnyrychovÁ, A multivalued version of Sharkovskiǔs theorem holds with at most two exceptions, J. Fixed Point Theory Appl. 2 (2007), 153-170. 
[11] Yu.G. Borisovich, B.D. Gel'man, A.D. Myshkis and V.V. OBukhovskiĬ, Topological methods in the fixed point theory of multi-valued maps, Russian Math. Surveys 35 (1980), 65-143.

[12] P. Minc and W.R.R. Transue, Sharkovskiu's Theorem for hereditarily decomposable chainable continua, Trans. Amer. Math. Soc. 315 (1989), 173-188.

[13] D. Repovs and P.V. Semenov, Continuous selections of multivalued mappings, mathematics and its applications, Kluwer Academic Publishers, 1998.

[14] _ Continuous selections of multivalued mappings, recent progress in general topology (II), North-Holland, 2002.

[15] J.J. Sanchez-Gabites and J.M.R. SAnjuRJo, Multivalued maps, selections and dynamical systems, Topol. Appl. 155 (2008), 874-882.

[16] J.M.R. SAnjurjo, Selections of multivalued maps and shape domination, Math. Proc. Cambridge Philos. Soc. 107 (1990), 493-499.

[17] A.N. Sharkovskil̆, Coexistence of cycles of a continuous map of a line into itself, Ukrai. Math. J. 16 (1964), 61-71. (Russian)

Manuscript received April 4, 2015

accepted May 5, 2015

JiEHUA MAI

College of Information and Statistics

Guangxi University of Finance and Economics

Nanning, Guangxi 530003, P.R. CHINA

E-mail address: jhmai@stu.edu.cn

TAIXIANG SUN

College of Information and Statistics

Guangxi University of Finance and Economics

Nanning 530003, P.R. CHINA

E-mail address: stxhql@gxu.edu.cn 